\title{
ROL DEL ENFERMERO/A RURAL EN LA REGIÓN DE LA ARAUCANÍA CHILE: PERCEPCIÓN DE USUARIOS Y ENFERMEROS
}

\section{ROLE OF THE RURAL NURSING IN THE ARAUCANÍA REGION OF CHILE: PATIENTS AND NURSES PERCEPTIONS}

\author{
Magdalena Marilaf C. \\ Ana M. Alarcón M. ** \\ Mónica Illesca P. ${ }^{* * *}$
}

\begin{abstract}
RESUMEN
La atención primaria rural ha tenido un desarrollo importante en los últimos años, acercando las acciones de los programas de salud a dicha población y aumentando considerablemente su nivel de resolución. Este estudio se propone descubrir la percepción de usuarios y enfermeros/as del sector rural respecto del rol del enfermero/a en las postas rurales de la Región de la Araucanía, Chile. Corresponde a un estudio cualitativo-descriptivo basado en las narrativas de informantes claves, desarrollado entre enero y diciembre del 2009, en las tres comunas con mayor índice de ruralidad del borde costero sur de la región. En él participaron 30 informantes; 27 usuarios de postas rurales (nueve usuarios por posta) y tres enfermeros/as, quienes atienden esos centros de salud. El análisis se efectuó mediante un proceso de segmentación, codificación, categorización, y síntesis conceptual de las narrativas recopiladas. Para asegurar el rigor del estudio se realizó triangulación de datos, de fuente informante y doble análisis. Los resultados muestran la existencia de 4 categorías en el reconocimiento del rol profesional: las funciones, imagen o representación a partir de sus acciones y continuidad, competencias profesionales concentradas en habilidades, conocimientos y destrezas; y finalmente la concepción del cuidado enfermero/a. Los resultados describen al enfermero/a rural parcialmente visualizado por usuarios, destacando la amabilidad, conocimiento, habilidad técnica y consejería como factores identitarios del rol. Mientras que los enfermeros/as se caracterizan por compromiso social, capacidad adaptativa, proactividad y manejo de tecnologías.
\end{abstract}

Palabras clave: Enfermería en salud comunitaria, rol profesional, competencia profesional.

\begin{abstract}
In Chile, primary healthcare has had an important development during last years carrying healthcare delivery to people in those places. This is a qualitative study based on informants' narratives whose objective is to discover the role of the nurse from the perspective of rural users, and nurses attending health rounds in three (3) coastal communities in the Araucanía Region from January to December 2009. Data used were obtained through in-depth interviews with 30 key informants. The analysis involved segmentation, coding, categorization, and conceptual synthesis of the narratives collected. Reliability addressed through triangulation of data, informant sources, and double analysis. The results showed four categories: role-based care and educational functions; image from his function and permanence, required competencies focused on knowledge and skills and finally, understanding of nursing care. The analysis describes the rural nurse partially, visualized by users, emphasizing their friendliness, knowledge, technical expertise and counseling as factors of identity of the role. On the other hand, nurses are characterized by social commitment, adaptive capacity, pro-activity and technology handling.
\end{abstract}

Key words: Community health nursing, professional role, professional competence.

Fecha recepción: 22.05.10 Fecha aceptación: 25.05.11

\footnotetext{
* Enfermera Cesfam Nueva Imperial. Programa Magíster de Enfermería Universidad de La Frontera Temuco, Chile. E-mail: magdaenf7@yahoo.es

** Enfermera. Profesora Dpto. Salud Pública Universidad de La Frontera. Temuco, Chile. E-mail: malarc@ufro.cl

${ }^{\star \star \star}$ Enfermera. Profesora Dpto. Medicina Interna Universidad de La Frontera. Temuco, Chile. E-mail: millesca@ufro.cl
} 


\section{INTRODUCCIÓN}

En Chile, las últimas décadas se han caracterizado por una mayor promoción y fortalecimiento del enfoque de salud familiar y de atención primaria en salud, situación que en el sector rural se ha traducido en una mayor asignación de recursos y equipamiento para las postas rurales (1). Asimismo, la Región de la Araucanía presenta la red más extensa de salud rural a nivel nacional constituida por 199 Postas y Estaciones Médico-Rurales (2). Estos centros son periódicamente visitados por un equipo multiprofesional, en el cual el enfermero/a cumple un rol fundamental tanto en la organización de los programas de salud, la gestión de las rondas rurales, como en la atención directa a la población rural. Así, el enfermero/a rural desarrolla un rol trascendental en la gerencia de los servicios y programas de salud, dado que aborda el proceso de salud desde el análisis y priorización de la situación hasta la evaluación y control de las acciones sanitarias (3). En consecuencia la definición, construcción y ejecución del rol del enfermero en tanto profesional de salud constituye un fenómeno de gran importancia para la salud rural.

Desde una perspectiva sociológica, el rol profesional se define como la construcción de una imagen social que se constituye en interacción con la comunidad o público, el cual perfila la identidad de este sujeto de acuerdo a su experiencia en ámbitos de sus necesidades (4).

El concepto de imagen se refiere a la figura, representación, semejanza o apariencia percibida por las personas respecto de este sujeto (5).

En este contexto, se señala que la imagen del enfermero/a es definida como una red de representaciones sociales en la que se involucran conceptos, afirmaciones y explicaciones sobre una practica de trabajo (6). De este modo, el rol es el conjunto de caracteres que hacen que alguien sea reconocido sin posibilidad de confusión con otro, lo que corresponde al imaginario o percepción que la población tiene del perfil del profesional $(7,8)$.

De este modo, la construcción de la identidad profesional implica un proceso dinámico entre un hacer y un percibir hacer, el cual está determinado por el contexto histórico, social, cultural, político y económico en que éste se desarrolla $(9,10,11)$.

Otro aspecto involucrado en la definición e identificación del perfil del enfermero/a corresponde al ejercicio de competencias profesionales, definidas como la combinación dinámica de atributos en relación a procedimientos, habilidades, actitudes y responsabilidades del sujeto, las cuales se organizan en genéricas y específicas $(12,13)$.

Las genéricas corresponderían a elementos comunes a cualquier profesión como por ejemplo capacidad de abstracción, análisis, síntesis, organización, planificación del tiempo, creatividad y comunicación; y entre las específicas se mencionan las habilidades interpersonales para trabajar en forma autónoma en la propia disciplina además de asumir compromisos éticos y de calidad de la profesión (14).

En Enfermería estas últimas se traducen en la capacidad de aplicar los conocimientos disciplinarios en el cuidado de la persona, familia y comunidad a través de todo su ciclo vital; utilizar tecnologías de información y comunicación para la toma de decisiones asertivas en el cuidado de salud, gestionar recursos; y planificar, organizar, ejecutar y evaluar actividades de promoción, prevención y recuperación de la salud con criterios de calidad y pertinencia cultural. Entre las habilidades propias de la disciplina se mencionan la aplicación del proceso de enfermería y teorías para organizar la intervención del cuidado, resolver los problemas de salud utilizando la investigación en enfermería, promover un proceso de aprendizaje permanente de las personas, grupos y comuni- 
dad, y promoción del autocuidado y estilos de vida saludable en relación con el medio ambiente (14).

Respecto a la enfermería rural se puede además señalar la capacidad de resolver problemas in situ en forma oportuna y eficiente, dado que la ruralidad constituye un importante factor de riesgo de acceso a los servicios; brindar cuidados integrales y continuos desde una perspectiva cultural, considerando que en el ámbito rural existen múltiples formas de cuidado cultural tradicional; y finalmente de gestión del acceso de la población a la red asistencial debido a que la posta rural es la puerta de entrada al sistema de mayor complejidad (15).

En nuestro país no existen estudios que profundicen específicamente en el rol del enfermero/a rural, en la imagen que la población ha construido de este profesional en base a sus acciones y competencias; o en la propia definición que los enfermeros/as rurales tienen de su rol profesional. Es por ello que este trabajo apunta a descubrir la percepción de los usuarios y enfermeros/as del sector rural en relación al rol del enfermero/a en las rondas de salud en las comunas del Sector Costero de la Región de la Araucanía.

Su propósito es aportar con conocimiento actualizado sobre un conjunto de definiciones profesionales que contribuyan tanto en la formación de enfermeros/as como en el desarrollo de la práctica disciplinaria.

\section{MATERIAL Y MÉTODO}

Corresponde a un estudio cualitativo-descriptivo basado en las narrativas orales de informantes claves. El estudio se desarrolló en las tres comunas con mayor índice de ruralidad del borde costero sur de la Región de la Araucanía (Carahue, Puerto Saavedra y Nueva Imperial). En el participaron 30 informantes, 27 fueron usuarios de postas rurales de las comunas antes mencionadas (nueve usuarios por posta) y tres fueron los enfermeros/as quienes atienden las postas rurales de dichas comunas. La opción de trabajar con informantes se basa en la necesidad de obtener información exhaustiva de sujetos que representan un conocimiento basado en su experiencia con el fenómeno en estudio. Los informantes en este caso contribuyeron a complementar las inferencias sobre el rol de la enfermería rural generada a través de la teoría. Entre los criterios de selección de los informantes usuarios se encuentran: antigüedad en el programa como indicador de haber generado una percepción más profunda tanto de las acciones como de sus prestadores; pertenecer o acudir a los programas de salud infantil, cardiovascular y adulto mayor en los últimos dos años en forma permanente, y cumplir con algunos requisitos propios de un buen informante, como por ejemplo disposición al diálogo, interés por enunciar su opinión y voluntad para entregar información referente a su experiencia con la enfermería rural (16). En relación a los informantes enfermeras/os se trabajó con el profesional que actualmente acude a las rondas rurales en las comunas seleccionadas. Los datos se recolectaron a través de sucesivas entrevistas en profundidad, grabadas previo consentimiento de los informantes. El estudio fue aprobado por el Comité de Ética Científico del Servicio de Salud Araucanía Sur. Estas fueron transcritas y analizadas bajo el esquema de análisis de contenido temático en cuatro grandes áreas: función del enfermero/a, imagen, competencias y concepto de cuidado en salud.

Para asegurar el rigor del estudio se realizó triangulación de datos a través de dos modalidades: a) fuente de informante ya que se escogieron tanto usuarios como enfermeros, los cuales aportaron una perspectiva émica y ética del rol en estudio, y b) de análisis, utilizando dos expertos independientes para el análisis de los textos narrativos $(17,18)$. 


\section{RESULTADOS}

Los resultados se organizan a partir de los cuatros temas centrales de investigación, los cuales han sido denominados categorías puesto que constituyen abstracciones conceptuales de las propiedades del objeto de estudio, en este caso el rol del enfermero/a rural. La siguiente figura representa un resumen general de los hallazgos, en ella se observa que en el rol del enfermero/a rural se distinguen cuatro categorías: la función ejercida tanto con el equipo de salud como con los usuarios, la imagen o representación del profesional, las competencias profesionales, y el concepto de cuidado en salud que sostiene a la disciplina.

Figura 1. Síntesis de la representación del rol del enfermero/a rural según la percepción de los informantes.

\begin{tabular}{|c|c|c|}
\hline \multirow{7}{*}{ Descripción del rol } & Categorías & Características/Propiedades \\
\hline & \multirow{3}{*}{ Función } & Asistencial \\
\hline & & Administrativa \\
\hline & & Educativa \\
\hline & $\begin{array}{l}\text { Imagen o representación } \\
\text { Profesional }\end{array}$ & $\begin{array}{l}\text { Reconocimiento de acciones y } \\
\text { continuidad. }\end{array}$ \\
\hline & Competencias profesionales & $\begin{array}{l}\text { Conocimientos, destrezas y } \\
\text { habilidades }\end{array}$ \\
\hline & Concepto de Cuidado en Salud & $\begin{array}{l}\text { Pertinencia y calidad de las acciones } \\
\text { del enfermero/a }\end{array}$ \\
\hline
\end{tabular}

\section{Función del Enfermero/a rural}

Las funciones identificadas por los informantes se agruparon según aquéllas internacionalmente establecidas en asistencial, administrativa y educativa (19).

\section{Asistencial:}

Se mencionan procedimientos tales como: "nos toman la presión, nos pesan y si estamos con sobrepeso nos derivan a la nutricionista, también a mí él mismo me ha hecho el electro". Se agregan a estos las curaciones, controles de salud en todas las etapas del ciclo vital, de salud infantil, del adulto mayor, cardiovascular, apoyo a pacientes discapacitados, terminales y a sus familias.

\section{Educativa:}

Se percibe como educador/a de los usuarios adultos en forma individual durante el control de salud y también en sesiones grupales programadas, abordando temas relacionados al cuidado de los niños/as con las madres, o autocuidado con los adultos mayores. A modo de ejemplo se señala: "enseñan cómo hay que vestirlos, cómo hay que tenerlo, cómo hay que cuidarlos, en su alimentación, en todo", "ahora estaba informando sobre las guagüitas, recién nacidas que para la estimulación de su desarrollo..."

Los enfermeros/as mencionan además la responsabilidad de capacitar al personal paramédico en procedimientos de enfermería y temas de actualidad. 


\section{Administrativa:}

Se describe a través de la realización de reuniones con el equipo o encargados de programas de la red asistencial; planificación de la ronda rural; recopilación, organización y consolidación de información estadística para dar cuenta del avance de los programas de Atención Primaria en Salud (APS): “Trabajo mucho con los paramédicos, porque todas las intervenciones que hacemos tienen que ser medibles, vamos a cumplir con objetivos, siempre los evaluamos, hacemos reunión por programas y evaluamos cómo hemos cumplido la calidad y metas que nos vamos proponiendo". Los enfermeros/as mencionan que algunas de estas actividades son realizadas fuera del horario laboral, como por ejemplo planificación de programas educativos e informes estadísticos.

Ninguno de los informantes mencionó elementos relacionados con el área de investigación en enfermería rural.

\section{Imagen o representación profesional del rol}

Los rasgos de identificación del profesional de enfermería según los usuarios apuntan primero al reconocimiento de sus acciones en el control de salud, "la que pesa, mide y saca electro"; el lugar físico donde el enfermero/a los atiende; y en último término a la identificación de un uniforme, por ejemplo: "a veces uno se guía por los delantales de la enfermera", "la que atiende aquí lleva una huinchita media azul", "la enfermera nunca lleva algo para distinguirla". Los enfermeros/as señalaron que se presentan como tal ante los usuarios; sin embargo indican que éstos no identifican claramente al enfermero/a dentro del equipo de salud: "todo el mundo está muy medicalizado, a todos le dicen doctor, excepto a las matronas", "muchos usuarios también llaman enfermera a los paramédicos, aunque creo conocen las diferencia de roles en cuanto a la atención"

\section{Competencias profesionales}

Se plantean como los conocimientos y habilidades propias del sujeto identificado como enfermero/a. Entre éstas cabe destacar los saberes sobre desarrollo, cuidado y crianza de los niños; cuidado y alimentación de los adultos mayores; atender y controlar enfermos; y proporcionar consejos al paciente y la familia. Una habilidad destacada por los usuarios fue la comunicación y amabilidad del profesional, junto con darse el tiempo suficiente para la atención. Las siguientes citas ejemplifican estos temas: "es amable, conversa con cariño", "él es como súper amoroso con los niños, no es de esos que son como cortantes, sabe explicar bien, los trata bien".

Los enfermeros/as consideran una competencia importante en el ámbito rural el tener conocimiento sobre la cultura del usuario mapuche como base para otorgar cuidados pertinentes en autocuidado, y conocer a las familias y comunidad. Un elemento muy ejemplificador de la adecuación del enfermero/a a la cosmovisión del usuario es el siguiente extracto: "Primero tienes que adaptarte a lo que ellos saben, incluso de repente me ha tocado gente que habla puro mapudungun y he tratado de que haya un intérprete, pero ahí, claro es de lo más complicado, por ejemplo hablo de la naturaleza, si le hablo de la sangre hablo del río, como que hago analogías con cosas naturales que ellos siempre van a tener, o que ellos conocen, porque yo no le voy a hablar del torrente sanguíneo".

\section{Concepto de cuidado en salud}

Este concepto emergió como un tema relevante durante las entrevistas, dado que constituye el pilar fundamental en la definición del rol enfermero/a rural. Los informantes representan este concepto con la idea de pertinencia, es decir cuidar significa para los usuarios "preocuparse de la salud de los niños o los ancianos", "preguntar por la fami- 
lia", "estar al tanto de lo que le pasa a uno"; y cuidado asociado a calidad representada por "atender bien", "escuchar los problemas", y "dar consejos buenos". En tanto los enfermeros/as identifican el concepto de cuidado basado en la noción de gestión del cuidado en la que involucran principalmente las funciones antes descritas (asistencial, educativa y de administración). Otro aspecto del concepto esta asociado a lo individualizado e integral, como por ejemplo lo mencionan las siguientes citas: "Debe ser integral, de acuerdo a lo que el paciente necesita no a lo que uno en realidad quiera darle, también considerando su cosmovisión, durante todo su ciclo vital en realidad", "un buen cuidado tiene que tener una continuidad en el paciente, ser cuidados continuos, integrales de acuerdo a la cosmovisión del paciente y al paciente mismo".

\section{DISCUSIÓN Y COMENTARIOS}

$\mathrm{Al}$ analizar los resultados se puede señalar que en las respuestas brindadas por los informantes usuarios y enfermeros/as se aprecia un importante consenso.

Llama la atención que en las funciones adscritas al rol enfermero/a éstos omitan la investigación como un aspecto a considerar dentro de las actividades a desarrollar por la enfermería rural. Algunos autores plantean esta situación como un desafío en la disciplina en general, demostrando que la mayor parte de las investigaciones publicadas provienen del mundo académico (20). Por otro lado en los usuarios el concepto de investigación es inexistente como una categoría del rol profesional. Para éstos el rol se define por el quehacer, como por ejemplo "controlar la presión y el peso, ver a los hipertensos, diabéticos, ancianos, visitar las casas, y pedir exámenes". En este aspecto, los enfermeros/ as reafirmaron las acciones mencionadas por los usuarios, señalando además que en algunas postas ejecutan procedimientos tales como curaciones avanzadas, toma de muestras y electrocardiogramas.

Otro elemento relevante en la identificación del rol del enfermero/a es su actividad educativa, la cual es percibida por los usuarios como un proceso de consejería o conversación que se otorga durante el control del paciente, abordando diferentes temáticas. Las personas valoran el consejo y la opinión del enfermero/a en el cuidado o recuperación de la salud, se percibe como una instancia de confianza y apoyo. A pesar de su importancia, los enfermeros/as indicaron cierta debilidad de esta función aludiendo a la falta de tiempo y dotación insuficiente de enfermeros/as rurales, valorando el apoyo que reciben de los internos de Enfermería y de los facilitadores interculturales, quienes permiten mejorar los procesos de comunicación con los usuarios.

En consecuencia, desde el punto de vista de las funciones propias de la enfermería, la imagen profesional se construye para la población rural desde la caracterización del hacer y el comunicar-educar.

Respecto de la identificación del enfermero/a dentro del equipo de salud, pocos usuarios nominan "enfermero/a" a quien identifican claramente por las funciones que desempeña, para la mayoría es el "doctor/a" o "señorita" que les realiza los controles y les aconseja, que atiende en un lugar fijo, y que le ofrece continuidad en la atención; sólo algunos mencionaron al uniforme como un elemento de identificación. Al parecer un tema subyacente en la construcción social del rol enfermero/a es el proceso de comunicación que se logra mediante un hacer dialógico con el usuario, en un contexto de continuidad de la atención, lo cual permite reconocer al profesional por el ejercicio de su rol. No obstante las postas rurales presentan una constante 
rotación de enfermeros/as, además de períodos prolongados entre un control y otro, situación que afectaría no sólo a la identificación del enfermero/a sino, lo que es más importante, a la salud de las personas.

Respecto de las competencias profesionales, es claramente mencionado en el estudio que el saber y el saber hacer caracterizan al enfermero/a, los usuarios reconocen al profesional por sus habilidades técnicas y conocimientos específicos sobre el desarrollo infantil y cuidados en salud. Los enfermeros/ as informantes señalaron además que la pertinencia cultural de las acciones y el conocimiento de la comunidad constituyen competencias necesarias para el trabajo rural, lo cual es particularmente importante para tener un mejor acercamiento a la población mapuche. A nuestro juicio, el manejo del modelo de enfermería intercultural debería ser un requisito básico para quienes trabajan en zonas rurales (21).

Finalmente, todos los temas tratados con los informantes nos convocaban a analizar el concepto de cuidado que sostiene la población como el eje que permite la construcción social del rol profesional. En este contexto se evidencia que para los usuarios el cuidar tiene una connotación de entregar atención con conocimiento, cariño, afecto y preocupación por el otro, situación que no es ajena a la opinión de los enfermeros/as, quienes a través del concepto gestión del cuidado sintetizan el rol como un saber hacer con pertinencia y continuidad.

Las descripciones presentadas en este estudio demuestran la importancia de distinguir y cuidar los saberes disciplinarios que sólo adquieren valor y significado en función de la interacción con la población, contribuyendo a la construcción de nuestro rol. Así, para el enfermero/a rural, es especialmente importante dar cuenta, en el poco tiempo de contacto con la comunidad, de una atención de excelencia en contenido y calidez.

\section{REFERENCIAS}

1. Sotelo J. La Visión de la OPS sobre la APS y recursos humanos en salud. [Internet]; 2008 Nov 4-6; Santiago, Chile: IX Reunión regional de los Observatorios en RHUS [citado 4 abril 2010]. 28 p. Disponible en http://www.redsalud.gov.cl

2. Colegio Médico de Chile. El Sistema de Salud Chileno. Contexto Histórico y Normativo. [Internet] año desconocido [citado 4 abril 2010]. 21 p. Disponible en http://www.colegiomedico.cl

3. Rojas C. El rol de la enfermera en el cuidado del paciente y en el desarrollo regional y del país. SITUA. 2004; 13 (2):24-44.

4. Netto L, Ramos F. Consideraciones finales sobre el proceso de construcción de la identidad del enfermero en el trabajo cotidiano. Rev Lat Am Enfermagen. 2004; 12 (1): 50-57.

5. RAE. [Internet]. Definición de conceptos. [citado 18 diciembre 2008]. Disponible en http://www.rae.es/rae.html

6. Silva AL, Padilha MI, Borenstein MS. Imagem e identidade profissional na construção do conhecimento em enfermagem. Rev Lat Am Enfermagem. 2002 10(4): 586-595.

7. Orozco L. El ser y quehacer de enfermería ¿qué hacer? Actual. Enferm. [Revista en Internet] 2005. [citado 15 febrero 2009]; 8 (1). Disponible en: http://www. encolombia.com/medicina/enfermeria/ Enfermeria8105-ser.htm

8. Cano-Caballero M. Enfermería y género, tiempo de reflexión para el cambio. Revista Index Enferm 2004; 13(46): 34-39.

9. Loo M. La identidad como proceso biológico-psicosocial y su construcción en enfermería. Rev Enferm IMSS. 2003; 11 (1): 49-54.

10. Lázaro E, Lavados S. Construyendo la Identidad Profesional de las Enfermeras 
a la luz de las representaciones sociales. V Jornada internacional, III Conferencia Brasileira sobre Representaciones sociales. [Internet]. 2007 [citado 10 abril 2010]. Disponible en http://www.vjirs. com.br/completos/VJIRS_0591_0687. PDF

11. Rodríguez-Camero ML, Rodríguez-Camero N, Azañón-Hernández R. La construcción mediática de enfermería. Index Enferm 2008; 17(2): 119-123.

12. Zabalza M. El trabajo por competencias en la enseñanza universitaria. [Internet]. año desconocido [citado 17 abril 2010]. 26 p. Disponible en http://www.upd.edu. $\mathrm{mx} /$ varios/simpdidac2007/Zabalza.pdf

13. Bravo NHB. Competencias Proyecto Tuning-Europa, Tuning, América Latina. [Internet] año desconocido [citado 10 abril 2010]. 27 p. Disponible en http:// acreditacion.unillanos.edu.co

14. Laka J, Narvaiza J. Universidades Chilenas. Proyecto Tuning. Competencias específicas, enfermería. Comparación con el conjunto de los resultados de América Latina. [Internet] Santiago, Chile: Observatorio Chileno de Políticas Evaluativas. FACSO; año desconocido [citado 11 abril 2010]. Disponible en http://www.opech. cl/educsuperior/superior_conocimiento/ educ_resultados_ce.pdf

15. Colegio de Enfermeras de Chile. Rol de la Enfermera. [Internet]. Santiago, Chile: Colegio de Enfermeras de Chile; año desconocido [citado 23 diciembre 2008]. Disponible en http://www.colegiodeenfermeras.cl

16. Hammersley M, Atkinson P. Etnografía, métodos de investigación. Barcelona: Paidós; 1970.

17. Morse JM (ed). Asuntos Críticos en los Métodos de Investigación Cualitativa. Antioquía: Editorial Universidad de Antioquía; $2003.447 \mathrm{p}$.

18. Polit D, Hungler B. Investigación Científica en Ciencias de la Salud. 6a ed. Madrid: Mc Graw-Hill Interamericana; 2000.

19. Consejo Internacional de Enfermeras. La Definición de Enfermería. [Internet] [Ginebra]: Consejo Internacional de Enfermeras; 2011 [citado 3 noviembre 2008]. Disponible en www.icn.ch/definitionsp. htm.

20. Alarcón AM, Astudillo P. La investigación en Enfermería en revistas Latinoamericanas. Cienc enferm. 2007; 13 (2): 25-31.

21. Leninger M. Transcultural Nursing: Concepts. Theories and Practices. Columbeus, Ohio. United Stated of America: Greyden Press; 1994. 